\title{
BMJ Open Efficacy and safety of butylphthalide for patients who had acute ischaemic stroke receiving intravenous thrombolysis or endovascular treatment (BAST trial): study protocol for a randomised placebo-controlled trial
}

\author{
Xuelei Zhang (10 , ,2,3 Anxin Wang, ${ }^{2,4}$ Jing Yu Zhang, ${ }^{1,2}$ Baixue Jia, ${ }^{1,2}$ \\ Xiaochuan Huo, ${ }^{1,2}$ Yingting Zuo, ${ }^{5}$ Xue Tian, ${ }^{5}$ Yilong Wang, ${ }^{2,4}$ Zhongrong Miao, ${ }^{1,2}$ on \\ behalf of the BAST study investigators
}

To cite: Zhang $X$, Wang A, Zhang JY, et al. Efficacy and safety of butylphthalide for patients who had acute ischaemic stroke receiving intravenous thrombolysis or endovascular treatment (BAST trial): study protocol for a randomised placebocontrolled trial. BMJ Open 2021;11:e045559. doi:10.1136/ bmjopen-2020-045559

- Prepublication history and additional supplemental material for this paper are available online. To view these files, please visit the journal online (http://dx.doi.org/10.1136/ bmjopen-2020-045559).

$\mathrm{XZ}$ and $\mathrm{AW}$ are joint first authors.

Received 05 October 2020

Revised 09 March 2021

Accepted 01 April 2021

Check for updates

(c) Author(s) (or their employer(s)) 2021. Re-use permitted under CC BY-NC. No commercial re-use. See rights and permissions. Published by BMJ.

For numbered affiliations see end of article.

Correspondence to

Professor Zhongrong Miao; 13601243293@163.com and

Professor Yilong Wang;

yilong528@gmail.com

\section{ABSTRACT}

Introduction As a neuroprotective medication, butylphthalide (NBP) may help protect against cerebral ischaemic injury. However, evidence on whether NBP influences the outcomes of patients who had acute ischaemic stroke who are receiving revascularisation treatment is limited. This study aims to evaluate whether additional NBP therapy can improve the functional outcome of patients who receive intravenous recombinant tissue plasminogen activator and/or endovascular treatment (EVT).

Methods and analysis The study will be a randomised, double-blind, placebo-controlled, multiple-centre, parallel group trial. The sample size is estimated at 1200 patients. Eligible patients will be randomised at a 1:1 ratio to receive either NBP or placebo daily for 90 days, which will include 14 days of injections and 76 days of capsules. The first use of NBP/placebo will be started within 6 hours of onset of ischaemic stroke. The primary outcome is the functional outcome as assessed by the 90-day modified Rankin Scale, adjusted for baseline scores on the National Institutes of Health Stroke Scale. The primary safety outcome is the percentage of serious adverse events during the 90 days of treatment. This trial will determine whether NBP medication benefits patients who had acute ischaemic stroke who receive intravenous thrombolysis or EVT.

Ethics and dissemination The protocol was written according to the general ethical guidelines of the Declaration of Helsinki and approved by the Institutional Review Board/Ethics Committee of Beijing Tiantan Hospital, Capital Medical University with approval number KY 2018003-02. Ethics committees of all participating sites have approved the study . Results of the study will be published in peer-reviewed scientific journals and shared in scientific presentations.

Trial registration number NCT03539445.
Strengths and limitations of this study

- The BAST trial (Butylphthalide for Acute Ischemic Stroke Patients Receiving Intravenous Thrombolysis or Endovascular Treatment) is a randomised, doubleblind, placebo-controlled, multiple-centre, parallel group study aimed to assess the efficacy and safety of butylphthalide (NBP) in patients who received intravenous recombinant tissue plasminogen activator and/or endovascular treatment.

- This is the first study to determine whether the application of NBP could improve functional outcome of patients who had acute ischaemic stroke receiving intravenous thrombolysis or endovascular treatment.

- Strict enrolment criteria, including the first injection started within 6 hours from onset, seem to be a challenge, which may result in bias between primary stroke centres and comprehensive stroke centres.

- The BAST study is conducted in China only, and the results may be limited to be extrapolated to other ethnic populations.

\section{INTRODUCTION}

Ischaemic stroke is the third leading cause of death globally due to its high prevalence, morbidity and mortality. ${ }^{1}$ Even if patients received standard intravenous recombinant tissue plasminogen activator (rt-PA) or endovascular treatment (EVT), a large proportion of patients cannot achieve functional independence. ${ }^{3-11}$ The ESCAPE-NA1 study (Efficacy and Safety of Nerinetide for the Treatment of Acute Ischaemic Stroke) showed that nerinetide had a therapeutic effect among patients who were not treated with intravenous thrombolysis. ${ }^{12}$ Although 
this finding required confirmation, it suggests that neuroprotection in human stroke might be possible. Since then, neuroprotective medicine has attracted the attention of clinicians, with the aim of reducing neuronal damage and improving neurological deficits.

Butylphthalide (NBP), which may have potential as a neuroprotective medication, is a family of compounds that have been isolated from the seeds of Apium graveolens Linn, of which the active ingredient is dl-3-N-NBP. NBP has been shown to reduce cerebral ischaemic damage and improve the clinical outcomes of patients. The underlying mechanisms have been confirmed in experimentation in animals and include promoting microcirculation $^{13}$; protecting blood-brain barrier ${ }^{14}$; releasing mitochondrial dysfunction ${ }^{15}$, and preventing poststroke inflammation $^{16}$ and cerebral oedema ${ }^{17}$. NBP has been approved for use in patients who had ischaemic stroke in China since 2002. Despite the recent development in reperfusion treatment for acute ischaemic stroke, it remains uncertain whether combination therapy with NBP improves patient outcomes.

This protocol describes the rationale and design of the BAST trial (Butylphthalide for Acute Ischemic Stroke Patients Receiving Intravenous Thrombolysis or Endovascular Treatment), which aims to investigate whether NBP is an effective and safe medication for patients who receive intravenous rt-PA and EVT.

\section{METHODS/DESIGN \\ Study design}

The BAST trial will be a randomised, double-blind, placebo-controlled, multiple-centre, parallel group study. It aims to assess the efficacy and safety of NBP in patients who receive intravenous rt-PA and/or EVT. Participants will be recruited from neurology or interventional neuroradiology departments from approximately 30 hospitals across China. Eligible patients will be randomised at a 1:1 ratio to receive either NBP or placebo daily for 90 days. They will be assessed on the day of randomisation, 2 days after the first injection, and on days 14, 30, 60 and 90 (table 1). The BAST trial design is in compliance with the Declaration of Helsinki. All patients or his/her legal representative will be asked to provide informed consent (see online supplemental file 2). The BAST study has been registered at ClinicalTrials. gov.

\section{Objective}

The primary objective of the trial is to determine whether administration of NBP improves the functional outcome of patients who had acute ischaemic stroke who receive intravenous thrombolysis or EVT.

\section{Participants}

All patients who arrive at the hospital presenting with sudden neurological function deficits will be recruited and screened for eligibility based on the inclusion and

\begin{tabular}{lllllll} 
Table 1 Visit plan & \multicolumn{1}{c}{ Day } & Day & Day & Day & Day \\
Measures & Baseline & 2 & 14 & 30 & 60 & 90 \\
\hline Demographics & $x$ & & & & & \\
History of present & $x$ & & & & & \\
illness & & & & $x$ & $x$ & $x$
\end{tabular}

\begin{tabular}{lllllll} 
mRS & $\mathrm{x}$ & $\mathrm{x}$ & $\mathrm{x}$ & $\mathrm{x}$ & $\mathrm{x}$ & $\mathrm{x}$ \\
History & $\mathrm{x}$ & & & & & \\
Medication & $\mathrm{x}$ & & $\mathrm{x}$ & $\mathrm{x}$ & $\mathrm{x}$ & $\mathrm{x}$ \\
NIHSS & $\mathrm{x}$ & $\mathrm{x}$ & $\mathrm{x}$ & & & $\mathrm{x}$ \\
Head CT & $\mathrm{x}$ & & & & & \\
Head MRI & & & $\mathrm{x}$ & & & \\
ASPECT & $\mathrm{x}$ & & & & & \\
$\begin{array}{l}\text { Laboratory } \\
\text { examination* }\end{array}$ & $\mathrm{x}$ & & $\mathrm{x}$ & & & \\
\hline
\end{tabular}

\begin{tabular}{|c|c|c|c|c|c|}
\hline Electrocardiograph & $x$ & & & & \\
\hline $\begin{array}{l}\text { Inclusion and } \\
\text { exclusion criteria }\end{array}$ & $x$ & & & & \\
\hline Informed consent & $x$ & & & & \\
\hline Randomisation & $x$ & & & & \\
\hline Injection & $x$ & & & & \\
\hline Compliance & & $x$ & $x$ & $x$ & $\mathrm{x}$ \\
\hline $\begin{array}{l}\text { Special laboratory } \\
\text { test }\end{array}$ & $x$ & $x$ & & & $\mathrm{x}$ \\
\hline TOAST classification & & $x$ & & & \\
\hline \multicolumn{6}{|l|}{ OCSP classification $x$} \\
\hline EQ-5D & & & $x$ & $x$ & $x$ \\
\hline MMSE & & $x$ & & & $\mathrm{x}$ \\
\hline MoCA & & $x$ & & & $\mathrm{x}$ \\
\hline Soft capsules & & $x$ & & & \\
\hline AE/SAE & $x$ & $x$ & $x$ & $x$ & $\mathrm{x}$ \\
\hline
\end{tabular}

*Including at least test of blood glucose, blood routine examination (count of platelet), and renal and liver function (alanine transaminase, aspartate aminotransferase and creatinine).

AE, adverse event; ASPECT, Alberta Stroke Program Early CT Score; EQ-5D, EuroQol 5D; MMSE, Mini-Mental State Examination; MoCA, Montreal Cognitive Assessment; mRS, modified Rankin Scale; NIHSS, National Institutes of Health Stroke Scale; OCSP, Oxfordshire Community Stroke Programme; SAE, serious adverse event; TOAST, Trial of Org 10172 in Acute Stroke Treatment.

exclusion criteria (box 1). The investigators fully inform the patient and/or legal representative of the equal opportunity to use the test medication or placebo, the prognosis of the disease, and the adverse reactions that can occur, which are ultimately weighed by the patient or their relatives. Patients or their legal representative will provide informed consent prior to enrolment.

\section{Randomisation}

The randomisation procedure will be carried out using a computer-generated code and permuted blocks. This allows eligible patients to be assigned to NBP or placebo 
Box 1 Inclusion and exclusion criteria for the BAST study

\author{
Inclusion criteria. \\ - Age $\geq 18$ years. \\ - Diagnosed with acute ischaemic stroke. \\ - Within 6 hours from symptom onset. \\ - Baseline NIHSS score ranging 4 from 25. \\ - Receiving intravenous rt-PA or endovascular treatment (including \\ intra-arterial thrombolysis and mechanical thrombectomy), or intra- \\ venous rt-PA bridging endovascular treatment. \\ - Signed informed consent.
}

Exclusion criteria.

- $\mathrm{mRS}$ score $>1$ at randomisation (premorbid historical assessment), ASPECT $\leq 6$ confirmed by preoperation CT scan and diagnosed with intracranial haemorrhagic diseases (including intracranial haemorrhage, subarachnoid haemorrhage, etc).

- Already use NBP or any drugs containing NBP between onset and randomisation.

- Appeared with dysphagia before randomisation.

- With history of coagulation disorders, haemorrhagic diathesis, neutropaenia or thrombocytopaenia.

- With history of chronic hepatopathy, liver or kidney dysfunction $(\geq 3 x$ upper limit of normal alanine transaminase or $\geq 2 \times$ upper limit of normal creatinine).

- With history of severe cardiopulmonary diseases judged by investigators.

- With history of bradycardia (heart rate $<60$ beats per minute) or sick sinus syndrome.

- Having severe non-cardiovascular comorbidity with life expectancy $<3$ months or failed to follow the study for other reasons.

- History of drug or food allergy, or known to be allergic to the composition of drugs in this study.

- Contraindications to digital subtraction angiography procedure, including severe allergy for contrast agent with or without iodine.

- Pregnancy or lactation, or childbearing women, with documented negative pregnancy test, but without reliable contraception.

- Incapable to follow this study for mental illness and cognitive or emotional disorders.

- Unsuitable for this study in the opinion of the investigators.

ASPECT, Alberta Stroke Program Early CT Score; BAST, Butylphthalide for Acute Ischemic Stroke Patients Receiving Intravenous Thrombolysis or Endovascular Treatment; mRS, modified Rankin Scale; NBP, butylphthalide; NIHSS, National Institutes of Health Stroke Scale; rt-PA, recombinant tissue plasminogen activator.

at a 1:1 ratio. Patients will be assigned a random serial number based on their time of enrolment and provided with the corresponding medicines, which are beforehand blind-covered. Both researchers and patients will be blind to the treatment.

\section{Procedures}

Eligible patients will receive adjunctive NBP/placebo treatment alongside standard intravenous $\mathrm{rt}-\mathrm{PA}$ and/or EVT. Patients in the experimental group will receive NBP and a $100 \mathrm{~mL}$ sodium chloride injection two times per day during the initial 14 days and soft $0.2 \mathrm{~g}$ NBP capsules three times per day from day 15 to day 90 . The control group will receive a $100 \mathrm{~mL}$ placebo injection two times per day during the initial 14 days and soft $0.2 \mathrm{~g}$ placebo capsules three times per day from day 15 to day 90 . The first NBP/placebo injection will be administered within 6 hours of onset of ischaemic stroke. Patients will be recommended to continue the injections for 14 days and for a minimum of 10 days. The capsule administration will be started the day following the final injection, and patients will be recommended to continue taking the capsules until day 90. Each injection will last for at least 50 min and will be administered 6 hours apart. Patients will be asked to take the capsules daily before meals and record medication administration, which will be checked by researchers. The steering committee will make recommendations for concomitant medications. All secondary preventive strategies, including antithrombosis and management of risk factors, will be followed according to guidelines. However, neuroprotective medications such as Human Urinary Kallindinogenase, edaravone and any ginkgo-containing injections will be prohibited.

\section{Outcomes}

Primary outcome

The primary efficacy outcome is the proportion of patients with a favourable outcome 90 days after randomisation. A favourable outcome ${ }^{18} 19$ will be defined as a score of 0 on the modified Rankin Scale (mRS) in patients with a baseline score of 4-7 on the National Institutes of Health Stroke Scale (NIHSS); an mRS score of 0-1 in patients with a baseline NIHSS score of 8-14; and an mRS score of 0-2 in patients with a baseline NIHSS score of 15-25.

We will perform a prespecified subgroup analysis to estimate the effects of sex, age, baseline NIHSS score, history of hypertension, diabetes, aetiological subgroups and use of EVT to determine the homogeneity of treatment effects in these subgroups.

\section{Secondary outcomes}

The secondary efficacy outcomes will include the difference value of the NIHSS scores between baseline and days 14 and 90; the cerebral infarction volume on day 14; the recanalisation rate within the first 24 hours of treatment; the percentage of symptomatic intracranial haemorrhage within the first 24 hours; recurrent symptomatic ischaemic stroke and vascular events during the 90 days of treatment; any vascular complications due to vascular events (recurrent symptomatic ischaemic stroke, myocardiac infarction or vascular death) on day 90; the life quality score estimated by EuroQol 5D on day 90; cognitive function estimated by the Mini-Mental State Examination and Montreal Cognitive Assessment scales on day 90; and rate of favourable outcome on day 14 .

\section{Safety outcomes}

The primary safety outcome is the percentage of serious adverse events during the 90 days of treatment, which include any event resulting in prolonged hospital time, permanent damage to the body system/organ, a lifethreatening condition or death. The secondary outcomes will include symptomatic intracranial haemorrhage 
during 90 days of treatment, total mortality between days 14 and 90, adverse events between days 14 and 90, and serious adverse events within the first 14 days of treatment.

\section{Power and sample size calculation}

According to a previous study, ${ }^{20}$ we predict that the rate of the 90-day favourable outcome (based on adjusted mRS scores) will be $60 \%$ in the experimental group and $50 \%$ in the control group. The test level will be set at 0.05 . To achieve $90 \%$ power and a significance level of 0.05 (twotailed), each group will require 550 patients. Assuming a dropout rate of 10\%, 600 patients will be required for each group, for a total of 1200 patients in the trial.

\section{Statistical analyses}

The primary analysis will be based on the intention to treat principle. Primary efficacy in the two groups will be compared using $\chi^{2}$ test, and logistic regression will be used to calculate the OR and $95 \%$ CI. Missing outcome data will be imputed using the last observation carried forward method. Significance will be set at 0.05 and all statistical tests will be two-tailed. Furthermore, when $50 \%$ and $75 \%$ of participants have completed follow-up, formal interim analyses of the primary outcome will be conducted to determine overwhelming efficacy or futility; in these cases, we will consider stopping the trial. Overwhelming efficacy will be estimated using the O'BrienFleming boundaries on the binary outcome of the 90-day favourable outcome, with corresponding significance levels of $0.003,0.018$ and 0.044 .

\section{Patient and public involvement}

Patients will not be involved in the development of the research question, selection of outcome measures, design of the trial, recruitment of participants or conduct of the trial. Results of the trial will be disseminated to study participants through direct consultation with a trial clinician at completion of the trial as well as through the publication of the results.

\section{Ethics and dissemination}

The protocol was written according to the general ethical guidelines of the Declaration of Helsinki and approved by the Institutional Review Board/Ethics Committee of Beijing Tiantan Hospital, Capital Medical University with approval number KY 2018-003-02. Ethics committees of all participating sites have approved the study and the names of all ethics committees can be found in online supplemental file 1 . Results of the study will be published in peer-reviewed scientific journals and shared in scientific presentations.

\section{DISCUSSION}

The BAST trial will be a phase III randomised controlled study. It will be carried out in a Chinese population. This trial will explore the efficacy and safety of NBP, a potential neuroprotective medication, for patients who had acute ischaemic stroke who receive intravenous rt-PA and EVT.
Several reports ${ }^{20-24}$ indicated that NBP may have a beneficial effect on patients with ischaemic stroke. A multicentre, randomised, double-blind and placebocontrolled study showed that the NBP treatment significantly improved the neurofunctional deficits and the two groups did not significantly differ in the rate of adverse events. ${ }^{25}$ A systematic review that included 21 randomised controlled trials reported that NBP improves neurological function after acute ischaemic stroke and appears to be a safe treatment. ${ }^{26}$ Another systematic review that included 12 randomised controlled trials reported that the combined use of NBP and standard anti-ischaemic stroke drugs was more effective than use of standard drugs alone. ${ }^{27}$ In this study, we will further explore the efficacy and safety of NBP in patients who receive intravenous rt-PA and/or EVT.

An in vitro experiment has demonstrated that NBP can protect endothelial cells against oxidative/nitrosative stress and subsequent cell death by enhancing hypoxia-inducible factor- 1 alpha expression. ${ }^{28}$ The protective effect of NBP on mitochondrial function has been demonstrated in early animal studies, which showed that NBP improves the activity of $\mathrm{Na}^{+} /$ $\mathrm{K}^{+}$-ATPase and $\mathrm{Ca}_{2}{ }^{+}$-ATPase in the mitochondria. ${ }^{29}$ NBP has also been found to prevent the occurrence of ischaemic stroke via the improvement of cerebral microcirculation in stroke-prone renovascular hypertensive rats. ${ }^{30}$ NBP administration ameliorated the reperfusion-induced brain damage via enhancement of hepatocyte growth factor and inhibition of Toll-like Receptor 4 (TLR4)/nuclear factor-kappa B (NF-kB) and proinflammatory cytokines in vivo and in vitro. ${ }^{31}$ Additionally, many recent studies have shown that treatment with NBP influences the level of proteins, such as caspase- 3 and caspase- 9 , in the execution phase of cell apoptosis. ${ }^{29}$ This finding offers a potential approach towards the prevention of further cellular death in the ischaemic penumbra. Above all, NBP protects against ischaemic cerebral injury through several mechanisms, which include alleviating oxidative damage, regulating mitochondrial dysfunction, improving microcirculation, and inhibiting apoptosis and inflammatory response. These NBP effects provide the theoretical basis of this study. We speculate that NBP will play a role in preventing ischaemia reperfusion injury after intravenous rt-PA and EVT, and that combination therapy will improve patients' functional outcomes.

One strength of this study is the requirement of $\mathrm{NBP} /$ placebo to be administered within 6 hours from onset of ischaemic stroke. Treatment with intravenous rt-PA is administered within 4.5 hours and EVT is administered within 6 hours. Therefore, in our trial, neuroprotective treatment will be synchronised with the recanalisation treatment. It is well documented that almost immediately after vascular occlusion occurs, ischaemic cerebral injury begins. Moreover, reperfusion injury after recanalisation may sometimes 
aggravate tissue damage. In most previous studies, ${ }^{21}$ neuroprotective medicine is administered within 48 hours of stroke onset. However, we will administer the neuroprotective treatment in the superacute ischaemic injury phase, which will enable us to demonstrate whether patients who receive combination therapy during this phase achieve a better functional outcome.

This study has some limitations. The sample size is estimated at 1200 patients, which is considered relatively small for a phase III intervention trial. Nevertheless, this conservative estimate will allow us to estimate the primary outcome parameter with sufficient precision. Strict procedures, such as first injection within 6 hours from onset, will be a challenge for patient selection and may result in bias between primary stroke centres and comprehensive stroke centres. Finally, the BAST study will be conducted in China only, and the results may be limited to generalise to other populations.

\section{TRIAL STATUS}

The current protocol is version 21.0 (12 December 2019). This trial is in the process of recruiting participants. The actual trial enrolment started on 1 July 2018. We expect to enrol the target sample size by September 2022 and plan to continue with follow-up until December 2022.

\section{DATA SAFETY AND MONITORING BOARD}

The data safety and monitoring board will monitor the progress of the study to ensure patient safety and the highest standards of ethics. Annual monitoring will be performed by an independent clinical monitor. Interim progress reports will be sent to the academic committee.

\author{
Author affiliations \\ ${ }^{1}$ Department of Neurological Intervention, Beijing Tiantan Hospital, Capital Medical \\ University, Beijing, China \\ ${ }^{2}$ China National Clinical Research Center for Neurological Diseases, Beijing Tiantan \\ Hospital, Capital Medical University, Beijing, China \\ ${ }^{3}$ Center of Stroke, Beijing Institute for Brain Disorders, Capital Medical University, \\ Beijing, China \\ ${ }^{4}$ Department of Neurology, Beijing Tiantan Hospital, Capital Medical University, \\ Beijing, China \\ ${ }^{5}$ Department of Epidemiology and Health Statistics, School of Public Health, Capital \\ Medical University, Beijing, China
}

\section{Correction notice This article has been corrected since it was first published.}

Acknowledgements We thank Edanz's editing services for its linguistic assistance during the preparation of this manuscript.

Collaborators The BAST study investigators.

Contributors All authors participated in the conception and design of the study. $X Z, A W, J Y Z$ and $Y Z$ are responsible for patient enrolment and data acquisition. AW, $\mathrm{YZ}$ and $\mathrm{XT}$ contributed to data analysis plan. BJ, XH, YW and ZM accounted for all aspects of the work in ensuring that questions related to the accuracy and integrity of any part of the work were appropriately investigated and resolved. XZ and AW prepared the first draft of the manuscript. All authors have read and approved the final version of the manuscript.

Funding The study was supported by grants from the National Key Technology Research and Development Program of the Ministry of Science and Technology of the People's Republic of China (2016YFC1301501) and Shijiazhuang Pharmaceutical Group dl-3-butylphthalide Pharmaceutical. The funder had no role in the design of the study and collection, analysis and interpretation of data and in writing the manuscript.

Competing interests None declared.

Patient consent for publication Parental/guardian consent obtained.

Provenance and peer review Not commissioned; externally peer reviewed.

Supplemental material This content has been supplied by the author(s). It has not been vetted by BMJ Publishing Group Limited (BMJ) and may not have been peer-reviewed. Any opinions or recommendations discussed are solely those of the author(s) and are not endorsed by BMJ. BMJ disclaims all liability and responsibility arising from any reliance placed on the content. Where the content includes any translated material, BMJ does not warrant the accuracy and reliability of the translations (including but not limited to local regulations, clinical guidelines, terminology, drug names and drug dosages), and is not responsible for any error and/or omissions arising from translation and adaptation or otherwise.

Open access This is an open access article distributed in accordance with the Creative Commons Attribution Non Commercial (CC BY-NC 4.0) license, which permits others to distribute, remix, adapt, build upon this work non-commercially, and license their derivative works on different terms, provided the original work is properly cited, appropriate credit is given, any changes made indicated, and the use is non-commercial. See: http://creativecommons.org/licenses/by-nc/4.0/.

ORCID iD

Xuelei Zhang http://orcid.org/0000-0001-6209-8187

\section{REFERENCES}

1 Yang G, Wang Y, Zeng Y, et al. Rapid health transition in China, 1990-2010: findings from the global burden of disease study 2010. Lancet 2013;381:1987-2015.

2 Feigin VL, Forouzanfar MH, Krishnamurthi R, et al. Global and regional burden of stroke during 1990-2010: findings from the global burden of disease study 2010. Lancet 2014;383:245-55.

3 National Institute of Neurological Disorders and Stroke rt-PA Stroke Study Group. Tissue plasminogen activator for acute ischemic stroke. N Engl J Med 1995;333:1581-8.

4 Hacke W, Donnan G, Fieschi C, et al. Association of outcome with early stroke treatment: pooled analysis of Atlantis, ECASS, and NINDS rt-PA stroke trials. Lancet 2004;363:768-74.

5 Wahlgren N, Ahmed N, Dávalos A, et al. Thrombolysis with alteplase for acute ischaemic stroke in the safe implementation of thrombolysis in Stroke-Monitoring study (SITS-MOST): an observational study. Lancet 2007;369:275-82.

6 Lees KR, Bluhmki E, von Kummer R, et al. Time to treatment with intravenous alteplase and outcome in stroke: an updated pooled analysis of ECASS, ATLANTIS, NINDS, and EPITHET trials. Lancet 2010;375:1695-703.

7 Berkhemer OA, Fransen PSS, Beumer D, et al. A randomized trial of intraarterial treatment for acute ischemic stroke. $N$ Engl $J$ Med 2015;372:11-20.

8 Goyal M, Demchuk AM, Menon BK, et al. Randomized assessment of rapid endovascular treatment of ischemic stroke. N Engl J Med 2015;372:1019-30.

9 Campbell BCV, Mitchell PJ, Kleinig TJ, et al. Endovascular therapy for ischemic stroke with perfusion-imaging selection. N Engl J Med 2015;372:1009-18.

10 Jovin TG, Chamorro A, Cobo E, et al. Thrombectomy within 8 hours after symptom onset in ischemic stroke. N Engl J Med 2015;372:2296-306.

11 Saver JL, Goyal M, Bonafe A, et al. Stent-retriever thrombectomy after intravenous t-PA vs. t-PA alone in stroke. N Engl $J$ Med 2015;372:2285-95.

12 Hill MD, Goyal M, Menon BK, et al. Efficacy and safety of nerinetide for the treatment of acute ischaemic stroke (ESCAPE-NA1): a multicentre, double-blind, randomised controlled trial. Lancet 2020;395:878-87.

13 HL X, Feng YP. Effects of 3-n-butylphthalide on pial arterioles in focal cerebral ischemia rats. Acta Pharmaceutica Sinica 1999;34:172-5.

14 Chong ZZ, Feng YP. Protective effect of butylphthalide on brain tissue after traumatic brain injury in rats. Chinese $J$ Pharmacol Toxicol 1999;13:194.

15 Xiong J, Feng YP. The protective effect of butylphthalide against mitochondrial inhury during cerebral ischemia. Acta Pharmaceutica Sinica 2000;35:408-12. 
$16 \mathrm{HL}$ X, Feng YP. Inhibitory effect of chiral butylphthalide on inflammation after focal cerebral ischemia in rats. Acta Pharmaceutica Sinica 2000;21:433.

17 JM li, Zhao YN, Xue CJ. Effects of dl-3n-butyphthalide on cerebral blood flow and brain edema after severe diffuse brain injury in rats. Chinese J Brain Dis Rehab 2012;2:23-6.

18 Johnston KC, Bruno A, Pauls Q, et al. Intensive vs standard treatment of hyperglycemia and functional outcome in patients with acute ischemic stroke: the shine randomized clinical trial. JAMA 2019;322:326-35.

19 Adams HP, Leclerc JR, Bluhmki E, et al. Measuring outcomes as a function of baseline severity of ischemic stroke. Cerebrovasc Dis 2004;18:124-9.

20 Cui L-Y, Zhu Y-C, Gao S, et al. Ninety-day administration of dl-3-nbutylphthalide for acute ischemic stroke: a randomized, double-blind trial. Chin Med J 2013;126:3405-10.

21 Yan H, Yan Z, Niu X, et al. Dl-3-n-butylphthalide can improve the cognitive function of patients with acute ischemic stroke: a prospective intervention study. Neurol Res 2017;39:337-43.

22 Ding Y, Gu Z, Zhai T, et al. Effect of butylphthalide on new cerebral microbleeds in patients with acute ischemic stroke. Medicine 2020;99:e21594.

23 Xue L-X, Zhang T, Zhao Y-W, et al. Efficacy and safety comparison of DL-3-n-butylphthalide and Cerebrolysin: effects on neurological and behavioral outcomes in acute ischemic stroke. Exp Ther Med 2016;11:2015-20.
24 Zhang C, Zhao S, Zang Y, et al. The efficacy and safety of DI-3nbutylphthalide on progressive cerebral infarction: a randomized controlled STROBE study. Medicine 2017;96:e7257.

25 Cui LY, Liu XQ, Zhu YC. Effects of dl-3-Butylphthalide on treatment of acute ischemic stroke with moderate symptoms: a multi-center, randomized, double-blind, placebo-control trial. Chinese J Neurol 2005;38:251-4.

26 Wang DR, Liu M, Wu B. DI-3-butylphthalide for acute ischemic stroke: a systematic review. Chinese $J$ Evidence-Based Med 2010;10:189-95.

$27 \mathrm{Xu} Z-\mathrm{Q}$, Zhou Y, Shao B-Z, et al. A systematic review of neuroprotective efficacy and safety of DL-3-N-Butylphthalide in ischemic stroke. Am J Chin Med 2019:47:507-25.

28 Li L, Zhang B, Tao Y, et al. DL-3-n-butylphthalide protects endothelial cells against oxidative/nitrosative stress, mitochondrial damage and subsequent cell death after oxygen glucose deprivation in vitro. Brain Res 2009;1290:91-101.

29 Abdoulaye IA, Guo YJ. A review of recent advances in neuroprotective potential of 3-n-butylphthalide and its derivatives. Biomed Res Int 2016;2016:5012341

30 Liu C-L, Liao S-J, Zeng J-S, et al. dl-3n-butylphthalide prevents stroke via improvement of cerebral microvessels in RHRSP. J Neurol Sci 2007;260:106-13.

31 Zhang P, Guo Z-F, Xu Y-M, et al. N-Butylphthalide (NBP) ameliorated cerebral ischemia reperfusion-induced brain injury via HGFregulated TLR4/NF- $\mathrm{KB}$ signaling pathway. Biomed Pharmacother 2016;83:658-66 